\title{
Pediatric Research Hospitalization: Its Meaning to Parents
}

\author{
Audrey T. MaCollum ${ }^{[7]}$ and A. Herbert Schwartz \\ Department of Pediatrics, Yale University School of Medicine, \\ Yale-New Haven Medical Center, and Yale Child Study Center, New Haven, Connecticut, USA
}

\begin{abstract}
Extract
Expectations and attitudes of parents of 140 children awaiting admission to a pediatric research center were examined in semistructured preadmission interviews. Parental conceptions of the purpose of hospitalization are summarized in table I. Even after discussion with the physician-investigator, parents of 61 children (Group A) failed to comprehend that hospitalization was for some research purpose. Although the expectations of parents and physicians could not be uniformly compared, it was established that, in many instances, purposes of the research had been explicitly described and that parents had utilized denial and repression in blocking these anxiety-producing communications. Parents of 60 children (Group B) viewed hospitalization as combining therapeutic and research aims. Parents of 9 (Group C) viewed the aim as primarily research. Parents of 6 (Group D) viewed the aim as pure research. Views of parents of 4 were unclassified.

A broad spectrum of fears associated with the concept 'research' was expressed by parents in Groups, B, C, and D. Children were viewed to be endangered by their disorders, and no alternative source of medical care was considered available. The idea of declining hospitalization seemed to be guilt-laden, and most of these parents thought consent was their only choice.

It was concluded from this study that if informed parental consent is sought, there are four questions on which the parents and physicians should concentrate: In what ways do the proposed studies constitute research as distinct from diagnosis and treatment? What is the nature of these studies? What physical and psychological risks are involved? Could alternate methods be developed to meet the child's medical needs?
\end{abstract}

\section{Speculation}

Systematic comparison of the motivations and expectations of physicians and parents both prior and subsequent to hospitalization of children for research purposes is proposed. Such a study should clarify the delineations between research and medical care and should elucidate some of the sources of parental misconceptions. In addition, a systematic study of the adaptation of children and their subsequent recovery from the stress experiences associated with research procedures is intended. Such a study should yield guidelines for appraising psychological risk with parents prior to eliciting consent and would also stimulate the development of special nursing techniques to safeguard and perhaps enhance the psychological well-being of the patients. 


\section{Introduction}

The proliferation of clinical research centers in the USA since 1961 has stimulated consideration of the legal and ethical aspects of research involving human subjects. Ethical codes outlining the conditions under which such research is permissible have been designed $[1,2,4]$. The concepts embodied in such codes, however, are abstract and elude precise or uniform definition. If the pediatric research patient is to be safeguarded effectively, theoretical consideration of concepts such as 'informed consent' should be concomitant with study of the motivation and expectation of the patient and/or his guardian. This preliminary study examines the meaning to parents of hospitalization of their children in the Children's General Clinical Research Center, Yale University School of Medicine (CGRG).

The CCRC is an autonomous six-bed unit to which children are admitted as patients of physicians on the medical school faculty. The studies planned for each patient are formalized in a written day-by-day research protocol, subject to approval by the Program Director and the Committee on Clinical Investigation. Since October 1966, each physician-investigator has reviewed his protocol with the parents of patients and obtained their written consent in advance of admission.

\section{Methods}

Between December 1, 1964, and December 1, 1967, the mothers of 140 patients were interviewed by one social worker prior to the child's admission. Thirty-three fathers participated also. Parents of acutely ill children admitted on an emergency basis from other divisions were not interviewed. Study patients showed growth deviation, metabolic disease, gastrointestinal and cardiac abnormalities, malignant tumors, and blood dyscrasias. Thirty-nine patients had potentially fatal illnesses. Forty-nine patients $(35 \%)$ were admitted by one investigator and $20(14 \%)$ by a second. The remaining $51 \%$ of patients were admitted by 19 other investigators. Forty-two patients resided in New Haven or adjacent townships, 95 resided in other areas of Connecticut and 3 resided in other states.

Each physician determined the nature of the conferences with the parents concerning admission of their children. Interviews with the social worker followed at variable intervals. Semistructured in nature, the interviews were designed to evaluate the preparation of parents and, indirectly, of children for admission. Parents were invited to express their expectations and concerns about the hospitalization, to appraise the child's probable adjustment to hospitalization in rela- tion to his current phase of psychological development and to consider ways in which they and professional staff might assist the child in adapting to hospitalization [3]. In addition, parents were asked such questions as: 'What do you believe to be the purpose of this admission? What do you hope will be gained from it?' The content of the interviews was recorded promptly and reviewed with the Program Director, the Assistant Director, the individual investigators, and the nursing staff. (It was made explicit to parents that the interview material might be shared with others on the staff. On occasions when parents requested that particular material be considered confidential, the request was honored.) These records were studied to determine what attitudes towards the concept 'research' were expressed by the mothers. Since less than one quarter of the fathers of this patient population were interviewed, their attitudes were not examined in this study. Estimation was made of the mother's level of intelligence by noting quality of concept formation, capacity for abstract thought, vocabulary, and ability to describe events and experiences. Sixty-one mothers were judged to be at a superior level, 72 at an average level and 7 at a limited level. It is probable that the selective process had eliminated most mothers of limited intelligence from the study group. Prior to meeting the investigator, many families had been referred by local physicians or clinics to the general pediatric clinic and, subsequently, to the specialty clinic attended by the investigator. Strong motivation, perseverance, comprehension of the child's needs, and a capacity to form productive relations with physicians were characteristics required to implement referral. Physicians exercised choice in selecting patients to participate in clinical research; apparent success in communicating with parents may have influenced this selection.

Since the interviews had not been specifically designed to explore the meanings of research, data in comparable depth and detail were not uniformly available. Since parental concepts concerning the purpose of hospitalization had not been systematically compared with those of the physician-investigators, our results are inconclusive. Future studies, however, are planned to define further the issues and problems related to pediatric clinical research.

\section{Results}

The outcome of the interviews is summarized in table I. Mothers of 61 patients (Group A) thought the purpose of hospitalization to be diagnostic and/or therapeutic. These parents had failed to understand why the medical care of their children would be under taken in a research center. Some had concluded: 'The 
doctor must know we couldn't afford tests like these if we had to pay for them'; 'Since the CCRC is small, maybe the children are watched more closely'; 'The CGRC is where the worst cases go'; 'That is where children go when the doctor doesn't know what's wrong with them.' Other parents did not perceive the CGRC as being significantly different from other divisions in this research-oriented teaching hospital.

Mothers of 60 patients (Group B) considered hospitalization to be for both clinical and research purposes. Twenty-two (Group $B_{1}$ ) thought it to be primarily therapeutic, but were in accord with the physician's secondary research aim. Each mother stated that her initial anxiety about the research had been allayed by the physician's reassurance that the research-motivated procedures would not harm her child. Many characterized physicians as people of integrity whose own parenthood would guarantee their good will towards children. This sense of trust reflected the belief that the child would experience neither physical nor psychological harm, although there had usually been explicit discussion with the physician concerning only the former.

An additional 22 mothers (Group $\mathrm{B}_{2}$ ) desired diagnosis and treatment for their children, but were fearful of the purpose of the research. One mother protested: 'I don't want the doctors to study hypoglycemia; I want them to study my child!' Another mother said: 'I hope that after A is in the hospital, the doctors won't indulge themselves.' This remark was representative of the concern of this group that because of scientific curiosity, physicians might perform unnecessary tests

Table I. Maternal concepts of the purposes of CCRC hospitalization

Maternal view of hospitalization

Number of maternal responses

A. Diagnostic/therapeutic 61

B. Diagnostic/therapeutic and research 60

1. Research secondary to care of child

2. Research incompatible with care of child

3. Research represented only hope of saving life

4. Research necessary to establish diagnosis

C. Primary research

6

D. Pure research

E. Unclassified that would induce severe and undue stress. One fearful mother declined the admission; another initially refused, but later consented because she could see no other way of having her child evaluated; a third explicitly limited her consent to procedures necessary for diagnosis and treatment. Thus, this was a mistrustful group in contrast to Group $B_{1}$.

Mothers of the 10 children in Group $B_{3}$ considered the purpose of hospitalization to be primarily therapeutic, but they knew that a 'research drug' was to be used in treating the potentially fatal illnesses of their children. Although they believed that the 'research' might possibly save the child's life, they feared that the drug itself might cause death. One mother reflected: 'Perhaps it would be better for $\mathbf{B}$ to die quickly from the drug than to have a long, lingering death from the disease.'

Mothers of the 6 children in Group $\mathrm{B}_{4}$ believed that only through research could the diagnosis of their children's illnesses be established. Stated one mother: 'When the doctors know what's wrong, you go to a regular ward; when they don't know, you go to a research center.' Another stated, 'When you have a rare condition, you go to a research center; there's no place else.'

Mothers of nine patients (Group C) viewed hospitalization to be primarily for research purposes, with little chance of direct benefit to their children. Two of these families refused the hospitalization; the teenage son of a third, aware of the purpose for hospitalization, went home soon after admission with parental consent. The mother of a fourth patient required psychiatric intervention for decompensation associated with the admission. The only mother free of manifest anxiety about the research was one who regarded her child as hopelessly impaired; she had vowed to free herself from self-reproach by 'leaving no stone unturned'.

Mothers of four patients (seen before the policy of admitted as normal controls, viewed hospitalization as being for pure research and offering no benefit to their children. All manifested intense anxiety, one initially withholding consent and two giving consent limited to a few studies in the protocol.

Mothers of four patients (seen before the policy of protocol review was established) expressed such perplexity concerning the reasons for hospitalization that their expectations could not be categorized. There had been only brief communication with the physician in a busy clinic; comprehensive discussion had been deferred until after admission.

It might be expected that a mother's capacity to understand the investigator's research aim would be related to her level of intellectual abilities. Examination, however, revealed no significant clustering of expectations (within the categories delineated above) 
according to intellectual classification. No significant clustering was related either to the etiology (genetic, congenital, or acquired) or prognosis of the child's disease.

Without available records of the conferences between investigators and mothers, it was difficult to determine post hoc what precise emphasis, if any, had been given to the research aims. Some investigators indicated to the social worker that the boundaries between therapeutic and research aims in particular studies were loosely delineated in their own thinking. In certain instances, the research aspects of the admission had been minimized to avoid arousing anxiety. In other cases, the research aim and design had been explicitly described, and it was clear that parents had utilized denial or repression in screening out these communications. To determine whether the 'style' of communication of particular investigators exerted major influence upon maternal expectations, the relation between the identity of the investigators and maternal expectations was therefore examined. The distribution of maternal expectations in relation to the eight investigators who admitted five or more patients is shown in table II.

No significant distribution of maternal expectations in relation to identity of investigator was demonstrated, although it appeared that investigators Nos.2, 6, 7, and 8 achieved limited success in communicating their research aims, whereas investigator No. 5 achieved uniform success.

To determine whether certain maternal expectations were associated with particular protocols, the distribution of expectations in relation to the most frequently used protocols was examined. No significant distribution was observed. Seventeen patients were admitted on a uniform protocol by investigator No. 1 . Nine mothers considered the admission to be for therapeutic purposes only (Group A), and 8 considered it

\section{Table II}

\begin{tabular}{lrrrcc}
\hline & \multicolumn{5}{c}{ Category of maternal expectation ${ }^{x}$} \\
\cline { 2 - 6 } Investigator & $\mathrm{A}$ & $\mathrm{B}(1-4)$ & $\mathrm{C}$ & $\mathrm{D}$ & $\mathrm{E}$ \\
\hline No. 1 & 24 & 21 & 3 & 1 & \\
No.2 & 14 & 6 & & & \\
No.3 & 4 & 3 & 3 & 1 & \\
No.4 & 2 & & 2 & 4 & \\
No.5 & & 8 & & & \\
No.6 & 4 & 1 & & & 4 \\
No.7 & 6 & & & & \\
No.8 & 4 & 1 & & & \\
\hline
\end{tabular}

1 As classified in table I. to be for therapeutic and research purposes ( 3 in Group $\mathrm{B}_{1}$ and 5 in Group $\mathrm{B}_{2}$ ).

Neither the intellectual capacity of mothers, the nature of the child's disease, the identity of the investigator, nor the nature of the research protocol appeared to exert significant influence upon maternal expectations. The sole variable of probable significance was the policy of mandatory protocol review prior to admission. Prior to existence of this policy, $69 \%$ of the mothers believed the purpose of hospitalization to be entirely diagnostic or therapeutic. Subsequently, only $19 \%$ presented this view. There was a slight redistribution of maternal expectations associated with investigators Nos. 1, 2, and 3; the proportion in Group A decreased, and the proportion in Groups B, C, and D increased. Maternal expectations associated with the 9 investigators who adnitted patients only after the review policy was established, including investigator No.5, were all classified within Groups B, C, and D.

\section{Connotations of the Concept 'Research'}

Although research was sometimes considered to be the only available source of hope, the term (with few exceptions) had stimulated or served as the focus of anxiety for parents in Groups B, C, and D. There was evidence that in parents in Group A, anxiety had sometimes produced unconscious denial of the meaning of research. The concept 'research' denoted many specific dangers.

Research hospitalization implied to some mothers that the child must already have been in grave danger from his illness: 'There must be something wrong that the doctors haven't told me about'; 'Research means that you have a very, very bad sickness or a sickness that lasts a long time'; 'The doctors must have decided that there is nothing more they can do for $\mathrm{C}$, so they might as well use her for research'; 'The worst cases got to "the federal building" '. This term reflected her awareness that the CCRC is supported by federalfunds.

For some mothers, research implied that the child would be exposed to new dangers, which included a broad spectrum of physical hazards. Many mothers feared that biological materials, drugs or physical procedures used in tests would afford dangers varying from transient illness to death. One couple imagined that during an EEG, probes would be stuck into D's brain, producing possible brain damage. Another parent believed that the physician might accidentally introduce lethal air bubbles into her child's vein while running tolerance tests. The term 'guinea pig' was recurrently associated with the word 'research'; it had for some the specific meaning of being cut up and mutilated. These mothers thought surgery in a research center represented the ultimate danger to their children. It is impressive that such fears were entertained 
even after the danger of physical risk had been discussed.

For some mothers, the dangers of research to their children were primarily psychological. It was feared that loss of personal privacy for the child and family would result; that the children would be viewed with curiosity, manipulated ad lib by physicians, and that their defects would be widely exposed. For some parents, 'research' implied being helpless and emotionally overwhelmed. One mother remarked that 'research' stimulated memories of an earlier hospitalization of her son: 'I saw him in bed, terrified. He was surrounded by eight doctors-they must have looked huge to him. Even though they didn't do anything to him but feel his lever, he must have thought they were going to. He was so frightened.'

It was often unclear whether such anxiety-laden thoughts of one's child being overwhelmed, mutilated, exposed, brain damaged, and even killed would also have been stimulated by hospitalization not involving research. The fears of many mothers, however, were heightened by their awareness that the CCRG is supported by federal funds, and that no charges are made to families. This fact often suggested that parental control over the child's well-being might be lost. One mother exclaimed: 'The doctors seem to feel that $\mathrm{E}$ belongs to science; it's as though they loan her to me between studies.' Some mothers were motivated to 'live-in' by their need to act as 'watchdogs' and insure that no procedures, except those to which they had consented, would be done. One mother wryly asked: 'The papers I signed protect the hospital. Who protects my child?'

\section{Motivation and Choice}

Only two mothers expressed a sense of altruistic gratification associated with the idea of research. Conversely, only three mothers refused the admission. One must therefore question why the many mothers who experienced anxiety concerning research hospitalization nonetheless gave their consent. A clue to their conscious motivation is contained in the rccurrent statement, 'I have no choice'.

This statement embodied at least three distinct but interrelated meanings. First, each mother feared that her child was endangered by his disorder. The dangers perceived ranged from those associated with degenerative or fatal illnesses to psychosocial problems associated with growth deviations. The physician's recommendation to hospitalize the child served to confirm the existence of danger.'

Second, mothers recognized a need to protect their children from these dangers. They expressed the wish to make restitution for the harm (genetic or experimental) that they believed they had caused their chil- dren, or the wish to avoid future self-reproach and reproach by the children for medical neglect. The idea of declining hospitalization that might be helpful to the child was too guilt-laden to be tolerable.

Third, mothers believed that there was no alternate source of medical care available, except in distant medical centers. Mothers had consulted the physicianinvestigator upon referral and thought him to be the regional authority on the child's disorder. Few mothers felt competent to question his judgment concerning the nature of studies and/or treatment recommended, or to dclineate between studies crucial for the child's well-being and studies performed to increase medical knowledge. Only rarely had mothers been offered the alternative of hospitalization in another division. Thus, when hospitalization in the CCRC was recommended, mothers experienced only the choice between refusal and consent. For the reasons cited, refusal was usually a psychological impossibility.

\section{Discussion}

There exists a consensus that informed consent of the subject or his guardian is an ethical prerequisite for research involving human subjects. Our data suggest that there are four questions that should be posed to the parents of pediatric research patients and, in some cases, to the patients if the consent is to be truly informed:

1. In what ways do the proposed studies constitute research as distinct from diagnosis and treatment?

2. What is the nature of these studies?

3. What physical and psychological risks are involved?

4. In what alternative ways can the child's medical needs be met?

Forty-four percent of the mothers (Group A) failed to understand that admission of their children to a research center was, in some respect, for research purposes, although the admission had been discussed with the respective physician-investigators. The maternal views concerning the purposes of admission were not systematically compared with those of the investigators, but it was established that, in many instances, the meaning of research hospitalization had been explicitly described. Thus, although parental consent was given, it could not be termed 'informed'.

Among the remaining mothers, with the exception of those in Group E, a research aim underlying hospitalization was recognized although expectations of the mothers were often divergent from those of the investigators. 'Research' was usually understood by mothers to be the study of disease, as distinct from the study and care of the individual child. It was believed that the 
primary commitment of an investigator might be to 'science' rather than to the child's wclfare, and that incompatibility might exist between research aims and the child's needs. These concepts were anxiety laden.

To the investigators, the term 'research' included: exhaustive diagnostic investigation of one child to identify a rare disorder; exploratory study of biophysiologic mechanisms in a group of children with comparable symptomatology; meticulous observation of the response of an ill child to a promising therapeutic agent; specific techniques of data collection. For parental consent to become truly informed, therefore, the question, 'In what ways do the proposed studies constitute research as distinct from diagnosis and treatment?' should be carefully examined by parents and investigators.

With respect to the question, 'What is the nature of the studies?' there was variation in the degree to which parents sought or were able to assimilate information. It was clear, however, that a conscientious attempt to procure informed parental consent could be selfdefeating if more information were given at one time than the parent and child, if he were present, could absorb. Descriptions of technically complex studies and biochemical processes may enhance a sense of mastery in some persons, but, in others, feelings of helplessness and confusion may ensue. If consent is elicited before parents have had adequate opportunity to assimilate the information given and to deal with any effects thus stimulated, subsequent expressions of anxiety and anger may be anticipated.

The question, 'What are the risks involved?' was considered crucial by most mothers. When unusual physical risk was absent, mothers had been offered reassurance, but this did not always allay anxiety. Explicit appraisal of psychological risk had rarely occurred. In this era of specialization, it is not to be expected that all clinical investigators would be attuned to the psychological impact of research procedures upon a child, nor would they have the training in child development necessary for such consideration. If one accords to mental health an importance comparable with that of physical health, however, this is an essential consideration. Will the development of an infant girl be adversely influenced if she experiences motor restraint for almost continuous urine collection over a onemonth period? Will a five-year-old boy with developmentally appropriate anxieties about bodily mutilation recover fully from the massive stress of being overpowered, restrained, and subjected to repeated, frequent, and sometimes painful venipunctures?

An international code [2] states: 'Clinical research cannot be legitimately carried out unless the importance of the objective is in proportion to the inherent risk to the subject.' Child development research has not yet yielded defunitive criteria for appraising psychological risk. It is difficult to compare uncertain psychological losses with expected physical gains; however, consideration of psychological risk can be as continuous as consideration of physical risk, if pediatric research centers include staff members or consultants trained in the psychological aspects of child development. Parents can be provided opportunities to participate in appraisal of such risks. Systematic study of the responses and subsequent adaption by children to stressful procedures may lead to the design of special nursing techniques that would safeguard the psychological development of children hospitalized for research purposes.

The results of this study suggest that if parental consent is to reflect active choice rather than helpless acquiescence, information concerning alternatives to research hospitalization should be made available to parents. In some instances, parents could perhaps be offered the alternative of hospitalization of their children in clinical divisions. It is often assumed that the opportunity for studies at no cost at a children's research center will be welcomed by parents. The lack of payment can represent to some a frightening loss of the capacity for self-determination and protective control over the child's welfare. In all cases, consideration of available alternatives might serve to sharpen the delineation between research and clinical study, and to make apparent the unique purposes of a research center.

\section{References and Notes}

1. Beecher, H.K. : Ethics and clinical research. New Engl.J. Med. 275: 1354-1360 (1966).

2. Human experimentation. Code of ethics of the World Medical Association. Declaration of Helsinki. Brit.med.J. 5402: 177 (1964).

3. McCollum, A.T.: Mothers' preparation for their children's hospitalization. Soc. Casework 48: 407415 (1967).

4. Mrtahell, R. G.: The child and experimental medicine. Brit.med.J. 5385: 721-727 (1964).

5. The authors wish to express appreciation to Jerome Grunt, M.D., CGRC Program Director, whose interest and cooperation contributed substantially to the study program.

6. The patients were studied in the Yale University Children's Clinical Research Center supported by USPHS Grant No. FR-00125-05.

7. Requests for reprints should be addressed to: Mrs. Audrey MaCollum, Research Associate in Social Work, Department of Pediatrics, Yale-New Haven Hospital, 789 Howard Avenue, New Haven, Conn. 06504 (USA). 